\title{
A Novel and Efficient Hybrid Model of Radio Multipath-Fading Channels in Indoor Environments
}

\author{
J. H. Tarng, Member, IEEE, Wen-Shun Liu, Yeh-Fong Huang, and Jiunn-Ming Huang
}

\begin{abstract}
This paper presents a novel and efficient hybrid model combining a two-dimensional (2-D) site-specific model and a statistical model to characterize multipath fading in indoor environments. The site-specific model describes the propagation effects of interior walls and building walls. The latter model characterizes the effect of scattering due to rough surface boundaries and/or randomly positioned scatterers such as furniture and personnel, which significantly affects small-scale fading. The hybrid model is computationally efficient since only the 2-D site-specific model is needed. In addition to accurately predicting mean field strength, the model can effectively quantify the relative mean contribution of diffused scattering with a factor $r$. The factor is also an effective index to quantify the cluttering strength of the propagation environment: 1) light-cluttering situation $r \leqq 0.35$ and 2) heavy-cluttering situation $r \geqq 0.65$. Some blind tests validate the effectiveness of the model. $A$ large amount of experimental data for 2.44-GHz radio at many different sites shows that a Nakagami distribution describes the fading distribution well.
\end{abstract}

Index Terms-Fading, field strength measurement, indoor environment, radio propagation model.

\section{INTRODUCTION}

$\mathbf{R}$ ADIO propagation in indoor environments has received extensive interest in recent years because WLAN and personal communications systems, using the ultra-high-frequency band as a basis, have been applied inside large office buildings. Characteristics of the indoor propagation channel must be further explored to provide a reasonable cell coverage and to quantify their capacity for supporting wireless digital communication as well. Therefore, related works have developed propagation models for narrow-band and wide-band transmissions [1]-[9] in a complicated indoor environment. A phenomenon commonly known as multipath fading may both seriously influence the transmissions and degrade the performance of a communication system operating inside buildings.

The severity of this disturbance depends on the specific physical properties. If the multipath channel is well characterized, transmitters and receivers can be designed to match the channel and to reduce the effect of the disturbance. Since in-building propagation depends not only on the floor layout and building structures, but also on randomly positioned scatterers around the transmitting or receiving antenna, the radio signal should not be modeled only by deterministic methods [10], [11].

Manuscript received July 7, 1999; revised February 25, 2002. This work was supported in part by the National Science Council of Taiwan under Project NSC 87-2213-E-009-148 and NSC 88-2213-E-009-110 and in part by the Ministry of Education and the NSC, R.O.C., under Project 89-E-FA06-2-4.

The authors are with the Department of Communication Engineering, National Chiao Tung University, Hsin-Chu, Taiwan, R.O.C.

Digital Object Identifier 10.1109/TAP.2003.809822
In light of the above analysis, our work presents a novel and efficient hybrid physical-statistical model to explore the fading statistics in indoor environments. The proposed model consists of a two-dimensional (2-D) site-specific deterministic model and a statistical model. The former model incorporates the large-scale specific floor layouts and construction features of a propagation site, which are digitized into computer programs and evaluates the signal at designated locations based on ray-tracing technique [7]-[9]. It includes the average path loss due to interior wall or furniture obstruction, which contributes to shadow fading. Furthermore, [9] employs the patched-wall model to describe floor layout in detail by using patches with different dielectric constants and sizes, thereby increasing the prediction accuracy of field strength. It is a deterministic method to yield the mean signal strength and describes the global variations of the signal. The latter model characterizes the small-scale cluttering effect due to rough surface boundaries and randomly positioned scatterers such as furniture and personnel, which are seldom addressed by previous investigations [12]-[13]. It can effectively quantify the relative mean contribution of the random scattering with a factor $r$. Therefore, the hybrid model can evaluate the mean signal as well as effectively characterize the small-scale fading. Furthermore, the measured fading statistics and mean field strength of a $2.44-\mathrm{GHz}$ radio propagation at various sites in buildings validate the proposed model.

\section{HYBRID MODEL}

In the hybrid model, the electric field after reception by the antenna is determined by a superposition of deterministic and random rays. It can be expressed as

$$
E_{r}=E_{d r}+E_{s r}
$$

where $E_{d r}=\vec{\rho}^{*} \bullet \sum_{i} \vec{E}_{d i}$ and $E_{s r}=\vec{\rho}^{*} \bullet \sum_{i} \vec{E}_{s i}$ with $\vec{\rho}^{*}$ being the complex conjugation of unit polarization vector of the receiving antenna. $E_{d r}$ is equal to the summation of deterministic ray fields due to the average or effective environment, which are computed by the site-specific model [8], [9]. $E_{s r}$ is a random field with zero mean, which describes the received field due to rough surface boundaries and/or randomly positioned scatterers.

\section{A. Site-Specific Model}

1) Ray-Tracing Technique: The ray tracing technique is used to trace the significant direct (if existed), reflected, and transmitted rays from a transmitter location to a receiver location. The transmitting unit sphere is decomposed into many 


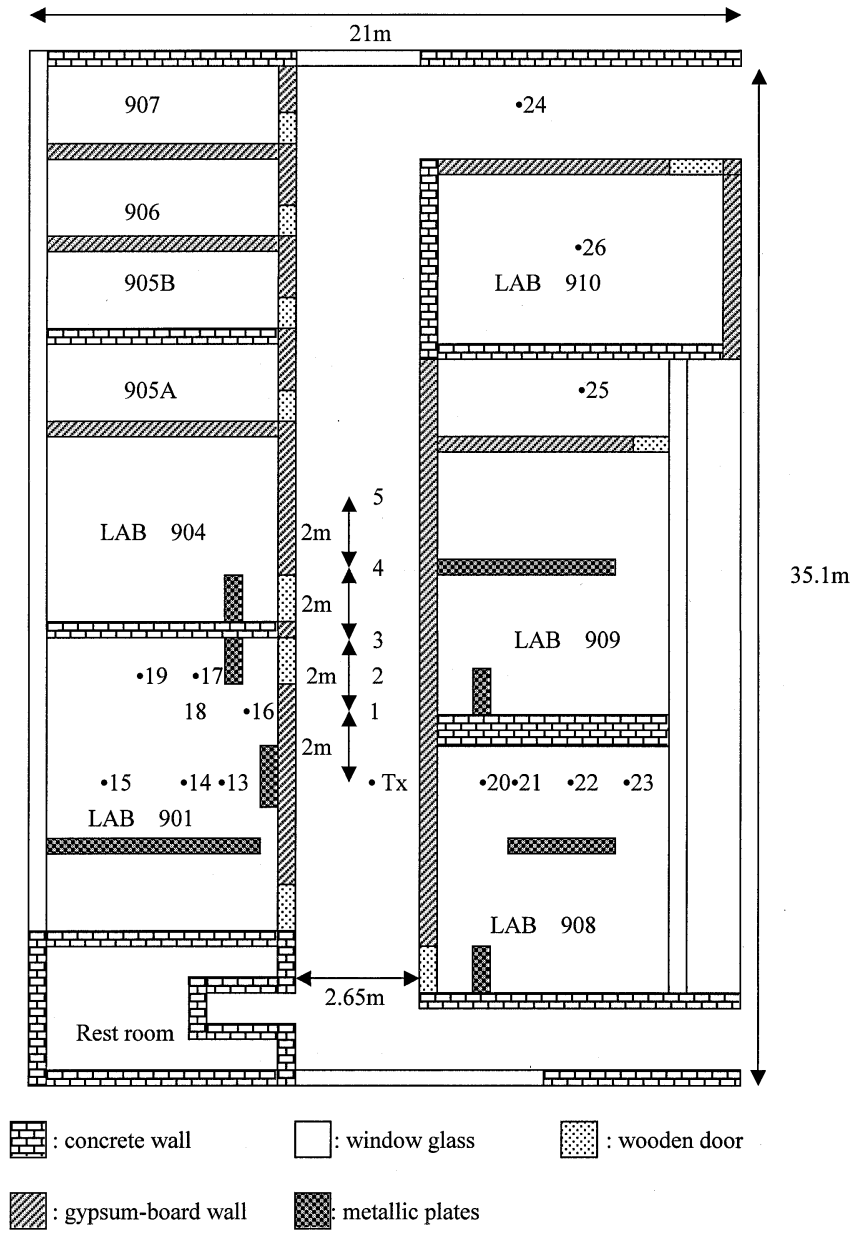

Fig. 1. Floor layout of sites A and C.

ray tubes and each is treated as a source ray during the tracing process. The tracing process is accomplished by an exhaustive search of a ray tree accounting for the decomposition of the ray at each building-object intersection. The reflected and transmitted rays are then treated separately in a similar fashion as a source ray. It is noted that the diffracted field is neglected.

2) The Site-Specific Model: The 2-D site-specific model includes direct, reflected, and refracted fields and describes them by rays [8], [9]. Each propagation mechanism is treated separately. The complex deterministic field $E_{d r}$ is given by

$$
E_{d r}=\sum_{i} E_{1 m} G_{t i} G_{r i} L_{i}(d) \prod_{j} R_{c}\left(\theta_{j i}\right) \prod_{m} T\left(\theta_{m i}\right)
$$

where $E_{1 m}$ is the field envelope $1 \mathrm{~m}$ away from the transmitting antenna; $G_{t i}$ and $G_{r i}$ are the field-amplitude radiation patterns of the transmitting and the receiving antennas, respectively; $L_{i}(d)$ is the free-space path loss of the $i$ th ray with an unfold length $d ; R_{c}$ and $T$ are the reflection and transmission coefficients, respectively; and $\theta_{j i}$ and $\theta_{m i}$ are the $j$ th and $m$ th reflecting and transmitting angles, respectively. Notably, in the computation of the reflection or transmission coefficient, the reflecting or transmitting boundary is treated as a multilayered structure and the ABCD matrix method is used [14]. In the line-of-sight (LOS) propagation, the floor-reflected ray is also included and computed by using (2) with $T=1$.

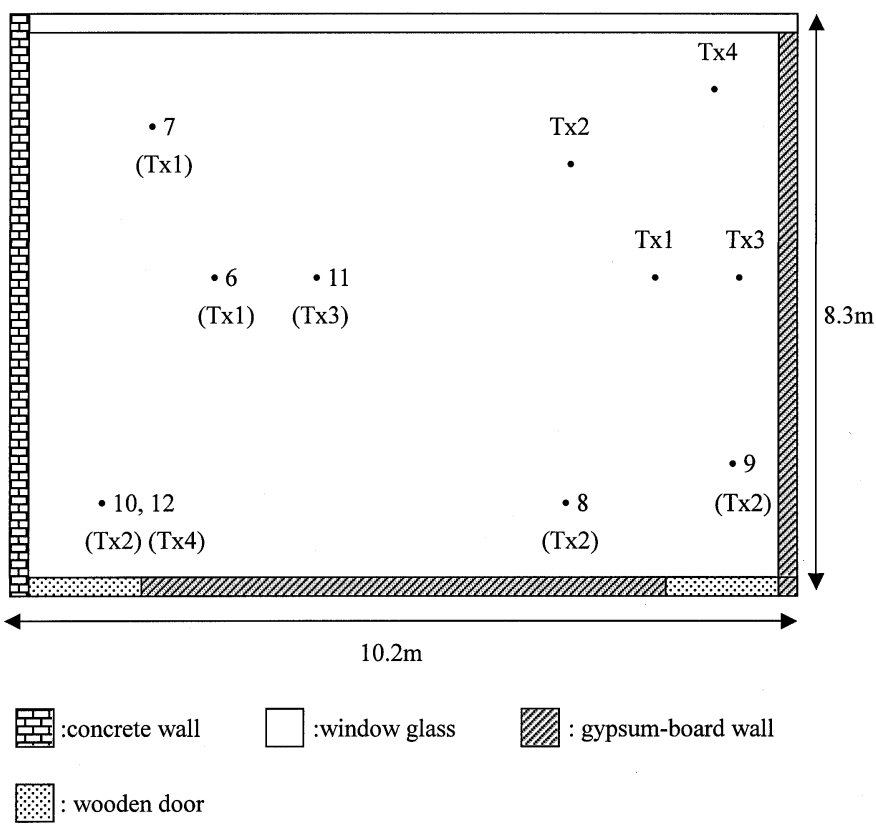

Fig. 2. Floor layout of site B. The receiving position number with bracket notation underline corresponds to the transmitting location.

\section{B. Scattering Model}

Previous investigations have proposed three models for mobile propagation environments to describe the scattered field [15], [16]. According to their results, these models are equivalent to each other as long as the total number of received rays is large enough. As is generally known, the coherence bandwidth of an indoor propagation channel is normally wider than the signal bandwidth of current communications systems, and the total number of received rays is large. Therefore, in this paper, one of these models is chosen to describe the randomly scattered field due to local scatterers inside buildings. With the chosen model, the angles of arrival were allowed to occur at random with equal probability for any direction. Although the phases were completely random, the amplitudes were assumed to be constant. Therefore, the receiving scattered field is formulated as

$$
E_{s r}=E_{0} \sum_{n=1}^{N} \exp \left(j \phi_{n}\right)
$$

where $\phi_{n}$ is a random phase that is uniformly distributed throughout 0 to $2 \pi ; N$ is the total number of received rays; and $E_{0}$ is an arbitrary constant. Notably, with statistical characteristics of the parameters in (3), $\left|E_{s r}\right|$ should follow a Rayleigh distribution. In addition, the spatial correlation function of the scattered field can be derived and given by

$$
R_{E_{s r}}(d)=\left\langle E_{s r}(r) E_{s r}^{*}(r+d)\right\rangle=N E_{0}^{2} J_{o}(k d)
$$

where $\langle\cdot\rangle$ and $J_{o}$ are the ensemble average and the zero-order Bessel function of the first kind, respectively; and $k$ and $d$ are the free-space wavenumber and spatial distance, respectively. In addition, the relation described in (4) will be used to generate the sampled scattered fields, which are correlated to each other. 

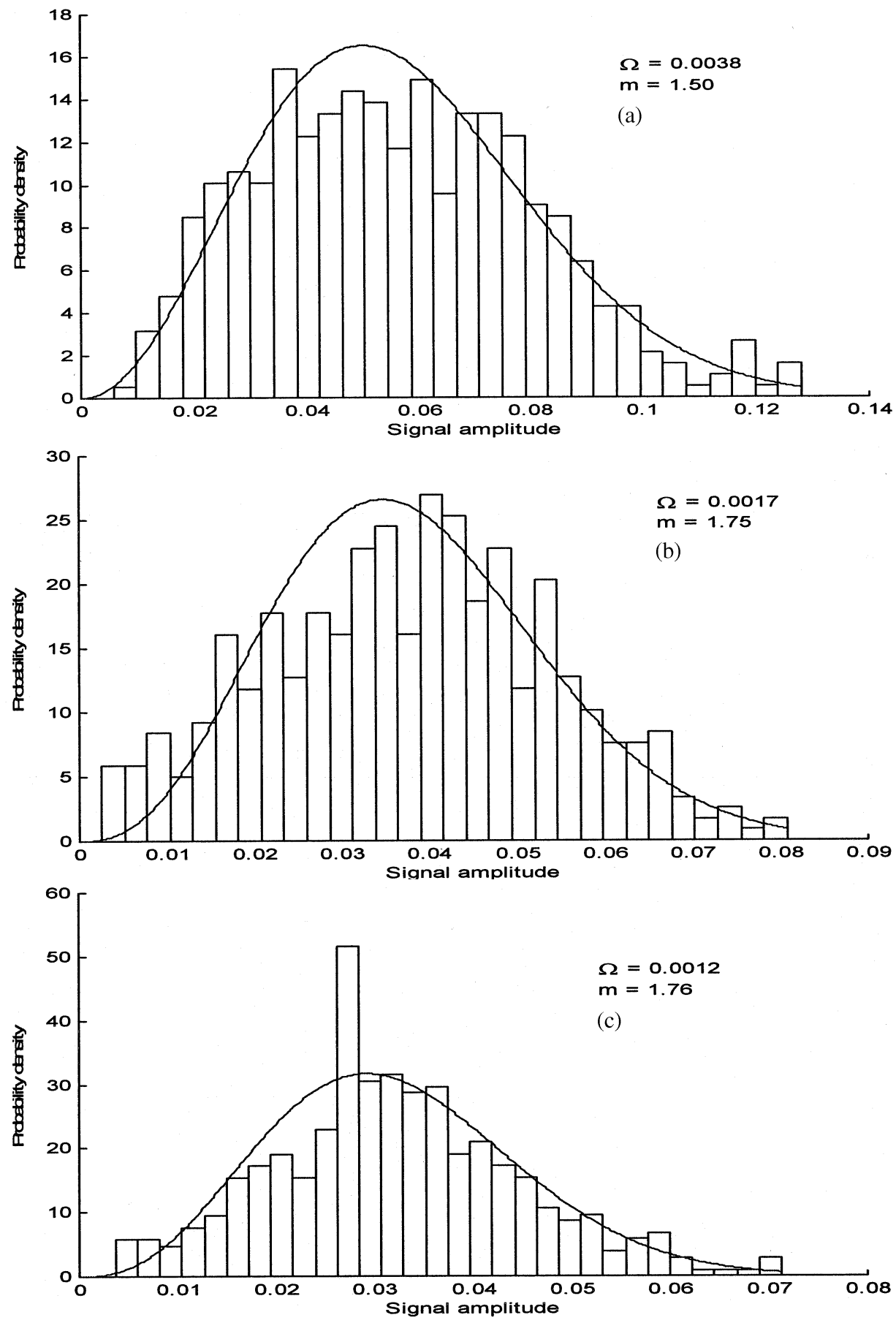

Fig. 3. Histogram and the best fit Nakagami distribution of signal amplitude for site A. (a) Receiving position no. 1. (b) Receiving position no. 2. (c) Receiving position no. 3 .

\section{Measurement Setup, Sites, AND Data Analysis}

\section{A. Measurement Setup and Sites}

Narrow-band (CW) signal strength measurements were taken at $2.44 \mathrm{GHz}$. A half-wavelength dipole antenna at a height $1.6 \mathrm{~m}$ above the ground transmits a $13-\mathrm{dBm} \mathrm{CW}$ signal. The transmitting system, including a signal generator, a section of cable, and the transmitting antenna, was calibrated in an anechoic chamber to measure the $1-\mathrm{m}$ transmitting field strength in free-space. The receiving antenna is also a half-wavelength dipole antenna (Anritsu MP663A) of the same height. Both the transmitting and receiving antennas are vertically polarized during the measurement. The receiver (Advantest R3261A) can instantaneously measure the signal strength between -30 to $-110 \mathrm{dBm}$ over a $100-\mathrm{kHz}$ interval. To ensure that the propagation channel is time stationary during the measurement, the measured data were averaged on screen over ten instantaneously sampled data. The received data were acquired automatically by a personal computer with a GPIB card. 
Each measured location was associated with a $5 \lambda \times 5 \lambda$ square area. In this area, $21 \times 21$-grid subpoints were chosen, and the received power was sampled at these subpoints. These subpoints were arranged in such a manner that 21 equally spaced subpoints were lined along each side of the square, i.e., $\lambda / 4$ spacing between any neighboring subpoints along each side.

There are 26 measured locations, all situated on the same floor of Engineering Building Number Four at the National Chiao-Tung University in Hsin-Chu. Both LOS and non-line-of-sight (NLOS) propagation measurements were taken. Measurement at site A (no. 1-no. 5), shown in Fig. 1, is LOS propagation in a corridor. It also displays site $\mathrm{C}$, where 14 receiving positions (no. 13-no. 26) are chosen and the direct path of each position is blocked (NLOS). At sites B, seven receiving positions are located at an empty room $(10.3 \times 8.4$ $\times 3 \mathrm{~m}^{3}$ ), as shown in Fig. 2, where four transmitting positions are chosen. These receiving positions are carefully chosen to include the generic propagation features.

To describe propagation environment at each site properly, the patched-wall model [9] is employed and five kinds of patch are used, including wooden doors, gypsum-board walls, window glass, concrete walls, and metallic plates. Four rectangular metallic patches are used to form the surfaces of the bookcase or the air conditioner. Metallic objects on the floor, including cabinets, bookcases, standing air conditioners, and whiteboards, are treated in the ray-tracing process since they are important reflecting articles.

\section{B. Measurement Data Analysis}

Fig. 3(a)-(c) display the histogram of the measured envelope at three receiving positions at site A. It is illustrated that the measured data are well described by the curve of the best-fit Nakagami distribution. The best-fit distribution implies that the parameters of the distribution such as the mean power $\Omega$ and the shape parameter $m$ are properly chosen to yield a minimum mean square error between the fitted and measured data. The Nakagami distribution has been shown to describe envelope fading well in multipath environments [10], [11]. It relates to other distributions through some simple formulas. For example, the Rayleigh distribution is a special case of the Nakagami distribution when $m=1$. The Nakagami distribution can closely approximate a Rician distribution. According to the comparison results in Fig. 3, the Nakagami distribution demonstrates a satisfactory performance in describing the fading envelope. For other receiving positions, their parameters of the best fit Nakagami distribution are summarized in Table I.

\section{COMPARISON AND ANALYSIS}

\section{A. Validation of the Site-Specific Model}

The measurement of the averaged path loss at $2.44 \mathrm{GHz}$ validates the 2-D site-specific model. In the numerical simulation made with the site-specific model, 360 source rays are generated and traced. The dielectric constants of gypsum-board walls, wooden doors, concrete walls, and ground floors are chosen to equal $5-0.062 j, 5-0.062 j, 7-0.6 j$, and $7-0.6 j$, respectively [17], [18]. Fig. 4 presents a scatter plot of the computed path loss versus the measured path loss at sites $\mathrm{A}, \mathrm{B}$, and $\mathrm{C}$. According
TABLE I

The Parameters of the Best Fit NaKagami Distribution at Sites A, B, AND C

\begin{tabular}{|c|c|c|c|}
\hline Site Number & Position Number & Measured $\Omega$ & Measured $\mathrm{m}$ \\
\hline \multirow{2}{*}{ Site A } & 4 & $6.84 \mathrm{e}-4$ & 1.92 \\
\hline & 5 & $3.93 \mathrm{e}-4$ & 1.2 \\
\hline \multirow{7}{*}{ Site B } & 6 & $1.96 \mathrm{e}-4$ & 1.80 \\
\hline & 7 & $2.04 \mathrm{e}-4$ & 2.00 \\
\hline & 8 & $3.87 \mathrm{e}-4$ & 1.94 \\
\hline & 9 & $4.03 \mathrm{e}-4$ & 1.34 \\
\hline & 10 & $3.34 \mathrm{e}-4$ & 2.08 \\
\hline & 11 & $2.84 \mathrm{e}-4$ & 1.76 \\
\hline & 12 & $1.88 \mathrm{e}-4$ & 1.67 \\
\hline \multirow{14}{*}{ Site C } & 13 & $2.38 \mathrm{e}-4$ & 1.19 \\
\hline & 14 & $1.31 \mathrm{e}-4$ & 0.97 \\
\hline & 15 & $1.38 \mathrm{e}-4$ & 1.62 \\
\hline & 16 & $5.42 \mathrm{e}-4$ & 1.10 \\
\hline & 17 & $4.35 \mathrm{e}-5$ & 1.36 \\
\hline & 18 & $9.69 \mathrm{e}-5$ & 1.10 \\
\hline & 19 & $1.02 \mathrm{e}-4$ & 1.44 \\
\hline & 20 & $3.32 \mathrm{e}-4$ & 1.13 \\
\hline & 21 & $2.56 \mathrm{e}-4$ & 1.07 \\
\hline & 22 & $1.07 \mathrm{e}-4$ & 1.38 \\
\hline & 23 & $1.52 \mathrm{e}-5$ & 1.28 \\
\hline & 24 & $4.19 \mathrm{e}-6$ & 1.10 \\
\hline & 25 & $8.18 \mathrm{e}-6$ & 1.11 \\
\hline & 26 & $1.97 \mathrm{e}-6$ & 1.33 \\
\hline
\end{tabular}

to this figure, both the 2-D site-specific and the hybrid models, which are combined with the patched-wall model, have a very good prediction accuracy. It is because of the small addition of the diffused term.

Notably, the number of ray-tree levels chosen in the ray-tracing process at each site must be greater than the maximum number of walls crossed by the direct ray between the transmitter and receiver. Here, nine tree levels are used to perform the simulations, which result in converged path loss.

\section{B. Computation of the Scattered Field}

Since the $(i+1)$ th sampled complex scattered field is related to the $i$ th sampled field, their relation can be described using first-order autoregressive model (AR-1) method [19]

$$
E_{s r}(i+1)=E_{s r}(i) R_{\rho}+\sqrt{1-R_{\rho}^{2}}\left[N_{1}(0, \sigma)+j N_{2}(0, \sigma)\right]
$$

where $R_{\rho}$ is the normalized $R_{E s r}$. Both $N_{1}(0, \sigma)$ and $N_{2}(0, \sigma)$ are independent Gaussian random variables with zero mean and standard deviation $\sigma$. Since $\left|E_{s r}\right|$ follows a Rayleigh distribution, $\sigma=\sqrt{2 / \pi}\left\langle\left|E_{s r}\right|\right\rangle$. Herein, the value of $\sigma$ or $\left\langle\left|E_{s r}\right|\right\rangle$ is determined from the experimental data. To determine $\sigma$ more easily, a factor $r=\left\langle\left|E_{s r}\right|\right\rangle /\left|E_{d r}\right|_{\text {ave }}$ is defined with $\left|E_{d r}\right|_{\text {ave }}$ being the spatially averaged deterministic envelope.

\section{Comparison}

Fig. 5 illustrates measured and computed probability density functions (pdfs) of signal amplitude of position no. 1 at site A. Both the site-specific and hybrid models are employed. Notably, 


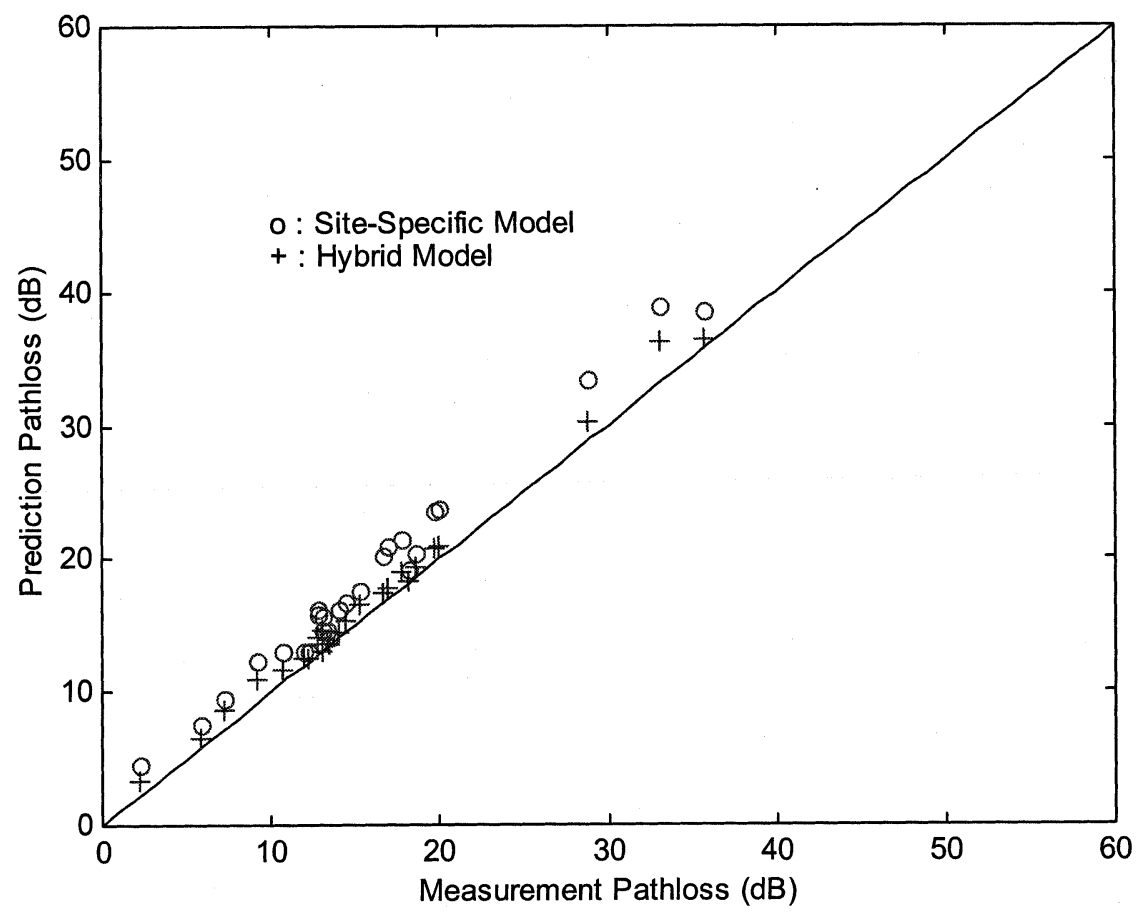

Fig. 4. A scatter plot of average path loss for sites A, B, and $\mathrm{C}\left(m_{e}=2.63 \mathrm{~dB}, \sigma_{e}=1.23 \mathrm{~dB}\right.$ for the site-specific model and $m_{e}=0.95 \mathrm{~dB}, \sigma_{e}=0.64 \mathrm{~dB}$ for the hybrid model).

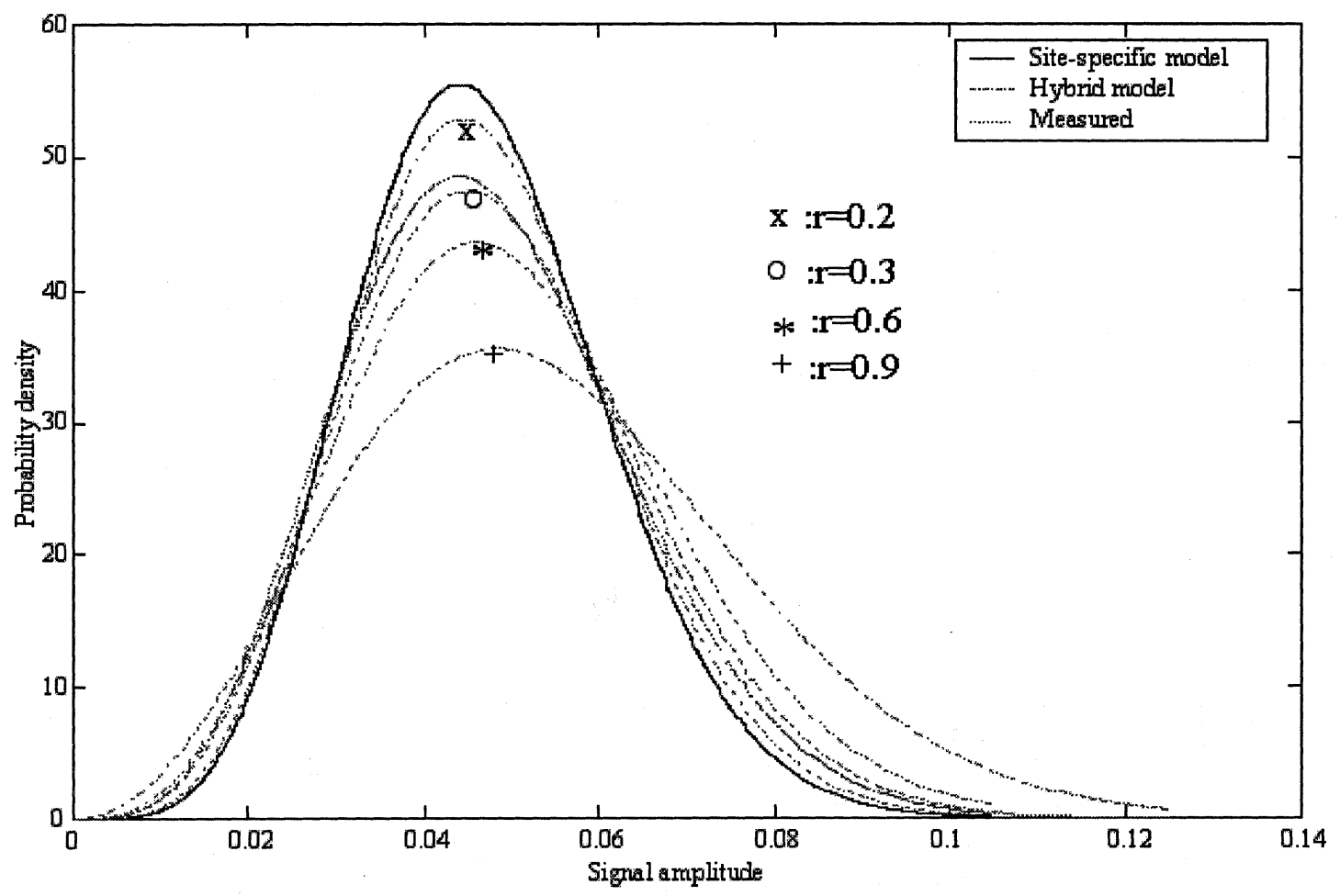

Fig. 5. PDF of measured and computed signal amplitudes of position no. 1 at site A.

the data computed by the hybrid model include the cases with $r=0.2,0.3,0.6$, and 0.9. From the figure, $r=0.3$ yields the best fit with the minimized mean square difference between the computed and measured pdf. It is also found that the hybrid model yields much better prediction accuracy than that of the site-specific model with a properly chosen value of $r$. Figs. 6 and 7 display measured and computed pdf of signal amplitude for a specific receiving position at sites $\mathrm{B}$ and $\mathrm{C}$, respectively. It is 


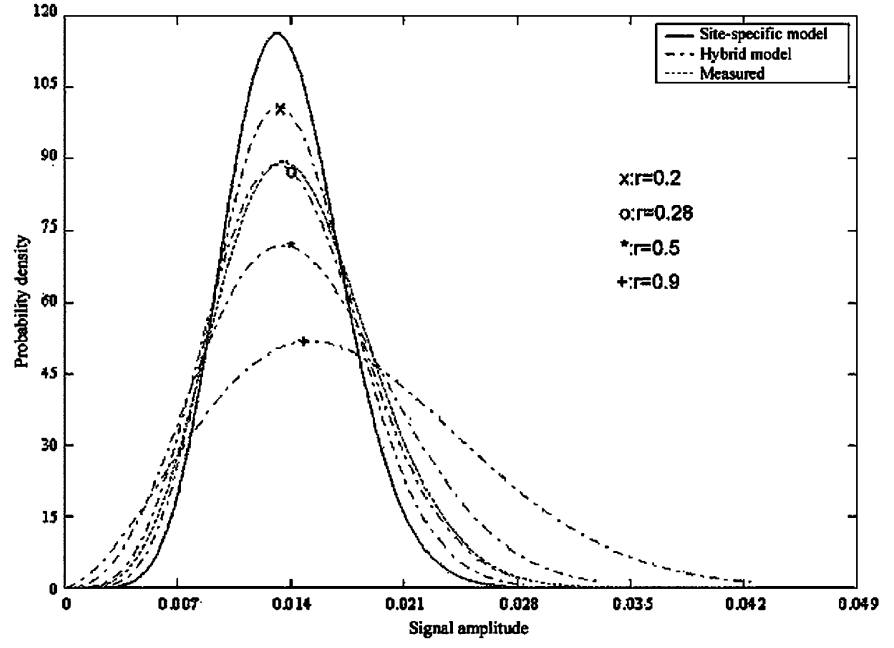

Fig. 6. PDF of measured and computed signal amplitudes of position no. 6 at site $\mathrm{B}$.

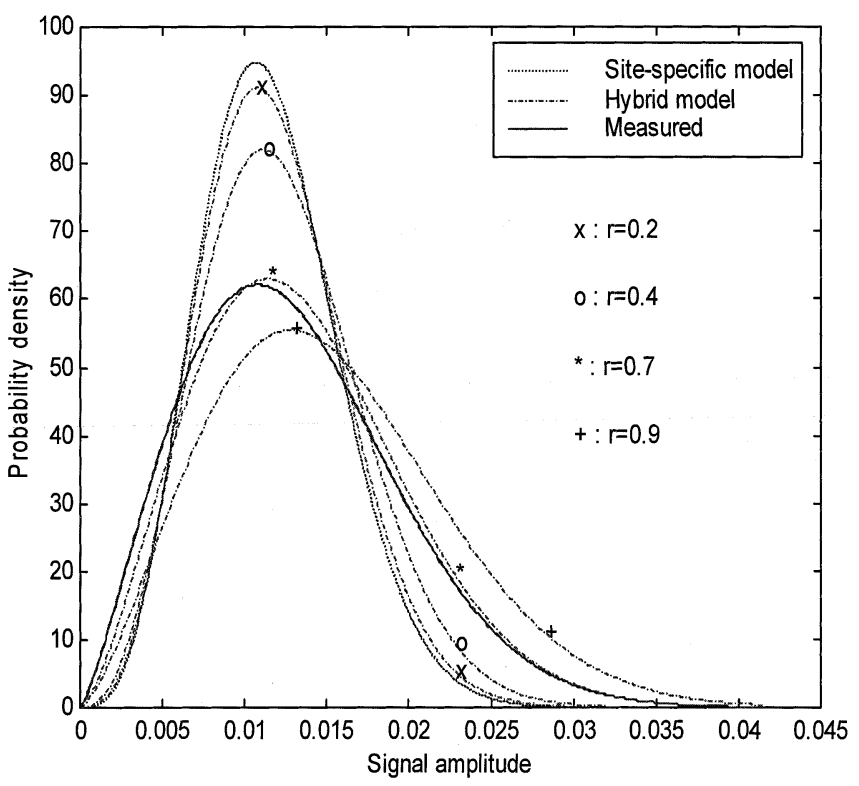

Fig. 7. PDF of measured and computed signal amplitudes of position no. 13 at site $\mathrm{C}$.

observed that the optimum values of $r$ for positions no. 6 and no. 13 are equal to 0.28 and 0.7 , respectively, and that the optimum value of $r$ may be varied with the propagation environment.

To learn the optimum range of $r$ in indoor environments, the root mean square error of the envelope's pdf $\sigma_{P D}$ for those 26 receiving positions are computed using different values of $r$. The mean of $\sigma_{P D}, \bar{\sigma}_{P D}$, versus $r$ is illustrated in Fig. 8. $\bar{\sigma}_{P D}$ is acquired by averaging over 10 samples of $\sigma_{P D}$.

In Fig. 8, it is found that the optimum value of $r$ varies in a range from 0.25 to 0.35 for light-cluttering situations and 0.65 to 0.75 for heavy-cluttering situations. This illustrates the fact that in heavy-cluttering environments where many scatterer clusters exist, the received amplitude fluctuates in wider range than in that of light-cluttering environments.

To find the optimum $r$ for indoor environments with a large open space, site D, the lobby of the Chung-Cheng Auditorium, is chosen for its large empty space $\left(19.7 \times 11.7 \times 12 \mathrm{~m}^{3}\right)$. Site
D has different propagation features from sites A, B, or C. Following the same procedure, the optimum $r$ for site $\mathrm{D}$ is small and equal to 0.2 .

Figs. 9 and 10 summarize the measured and computed $m$ and $\Omega$, respectively, for the 43 receiving positions. From Fig. 9, it is observed that the hybrid model yields a much better characterization accuracy of $m$ than that of the site-specific model, i.e., the proposed model can characterize the small-scale fading well. Both models have similar prediction accuracy of the mean power.

\section{Blind Test}

To validate the applicability of the hybrid model, it is verified by the measured data acquired at other four sites, sites E-H at different buildings on the campus. Layouts of these sites will not be illustrated in this paper in the interests of space. Site $\mathrm{E}$ is on the sixth floor of Graduate Student Dormitory no. Two. There are six receiving positions located at different rooms or in the corridor while the transmitter is situated in a room alone. The direct path does not exist. Site $\mathrm{F}$ is located in a computer room in Engineering Building no. Four. There are six receiving positions in the room, which is filled with computers and furniture. The transmitter is placed outside of the room. Site $\mathrm{G}$ is located in an empty classroom in Engineering Building no. Four. Site $\mathrm{H}$ is situated in the Gym, which has a large open space $(40 \times 34 \times$ $10 \mathrm{~m}^{3}$ ). Only one receiving position is arranged again, with the propagation distance being equal to $10 \mathrm{~m}$. The total number of the measured positions at these sites is equal to 14. Notably, $r$ for sites $\mathrm{E}-\mathrm{H}$ is determined by averaging the optimum range of $r$ obtained at sites $\mathrm{A}, \mathrm{B}$, and $\mathrm{C}$, i.e., $r=0.3$ for light-cluttering environments and $r=0.7$ for heavy-cluttering environments. For site $\mathrm{H}$, a large open area, $r=0.2$, which is the same as that at site D. In Figs. 9 and 10, position numbers greater than 26 are used for blind test. There is a dashed straight line as the boundary to split these two kinds of position, which is shown in Figs. 9 and 10. It means that the measured data of position number 1-26 calibrate the $r$ factor. From Figs. 9 and 10, it is found that the hybrid model using the average $r$ still yields a satisfactory performance at every receiving position. This test validates the proposed model.

\section{DISCUSSION}

In the following, we analyze the factors that affect " $r$," including wall roughness, single/multiple scatterer cluster, kind of room, LOS/NLOS, and propagation distance.

\section{A. Wall Roughness}

The effect of wall roughness in the buildings, including the effects of inhomogeneous wall structure due to the embedded windows, is discussed here. At site B, which is an empty room as shown in Fig. 2, $r$ is in the range of $0.27 \sim 0.3$ when the transmitting and receiving antennas are not close to the wall boundaries. Another experiment in a new empty room with a similar size, site $\mathrm{G}$ at the same building, is conducted; the room layout is shown in Fig. 11. It is found that $r=0.3$ (when the single scatter cluster does not exist), which is close to the value found at site B. It is also found that as the empty space increases, the 


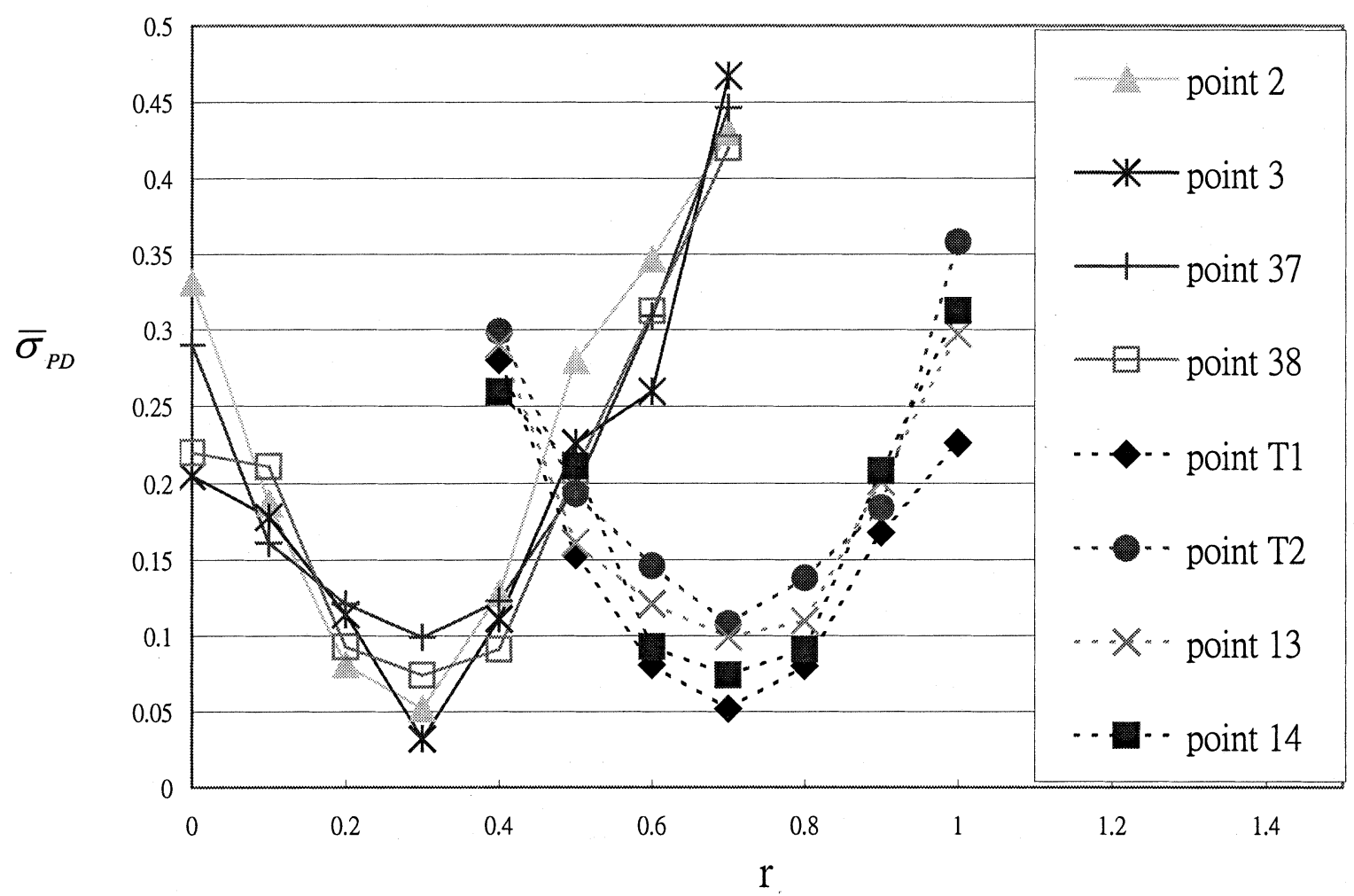

Fig. 8. Mean of $\bar{\sigma}_{P D}$ for eight sampled points illustrated as a function of $r$. It is observed that optimum $r$ is in the range of $0.2 \sim 0.4$ for the case of light cluttering such as at measured points $2,3,37,38$, and optimum $r$ is in the range of $0.7 \sim 0.8$ for the case of heavy cluttering such as at measured points 13,14 , T1, T2.

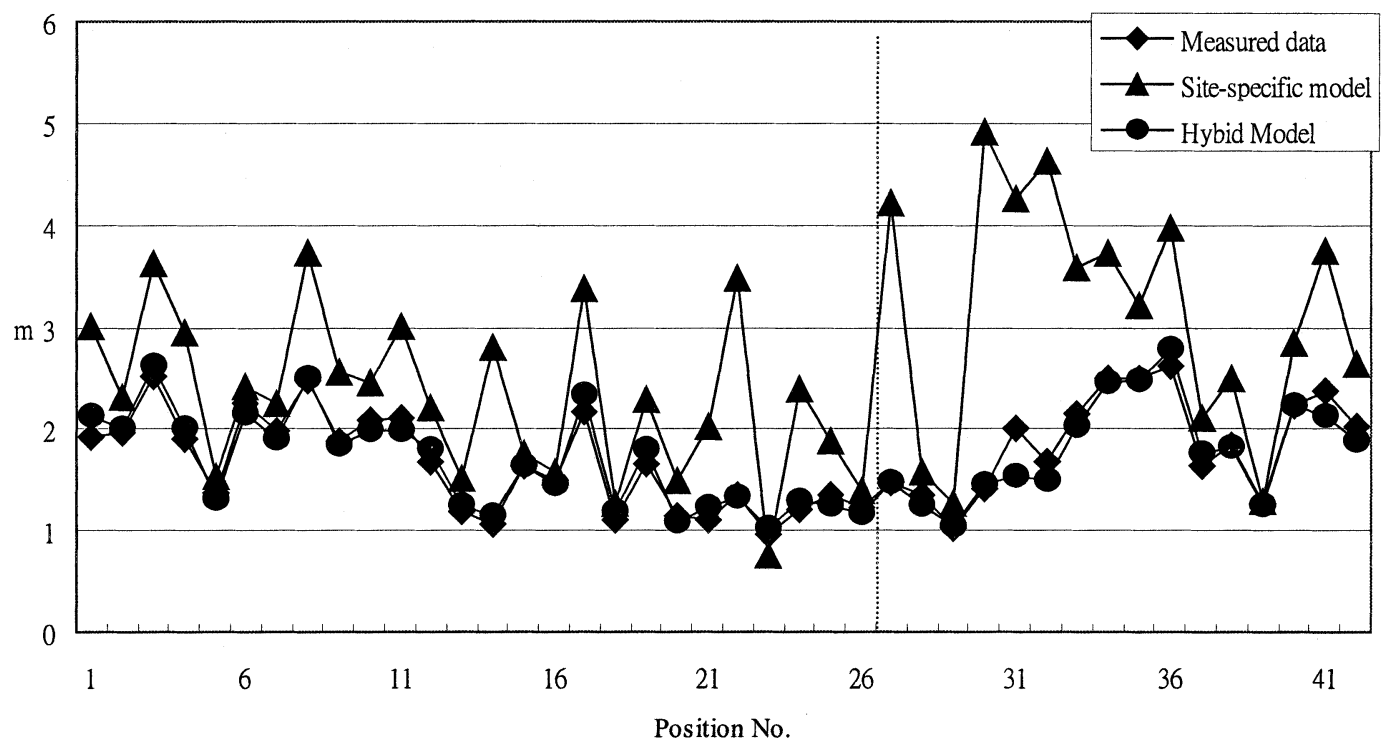

Fig. 9. The measured and computed $m$ are summarized for the 43 receiving positions, where $m$ is the shape parameter of Nakagami distribution. The measured data of position numbers greater than 26 are used for blind test. The hybrid model yields good prediction accuracy.

scattering effect of wall roughness decreases, i.e., $r$ decreases. It is observed that $r=0.2$ at point no. 43 at site $\mathrm{D}$, a large empty lobby. Therefore, wall roughness may affect $r$, but its effect decreases as both the receiving and transmitting antennas are moved away from the wall.

\section{B. Single/Multiple Scatterer Cluster}

To investigate the effect of a single scatterer cluster, a measurement is carried out in an empty room, site G, where many desks and chairs are piled up to simulate the scatterer cluster as 


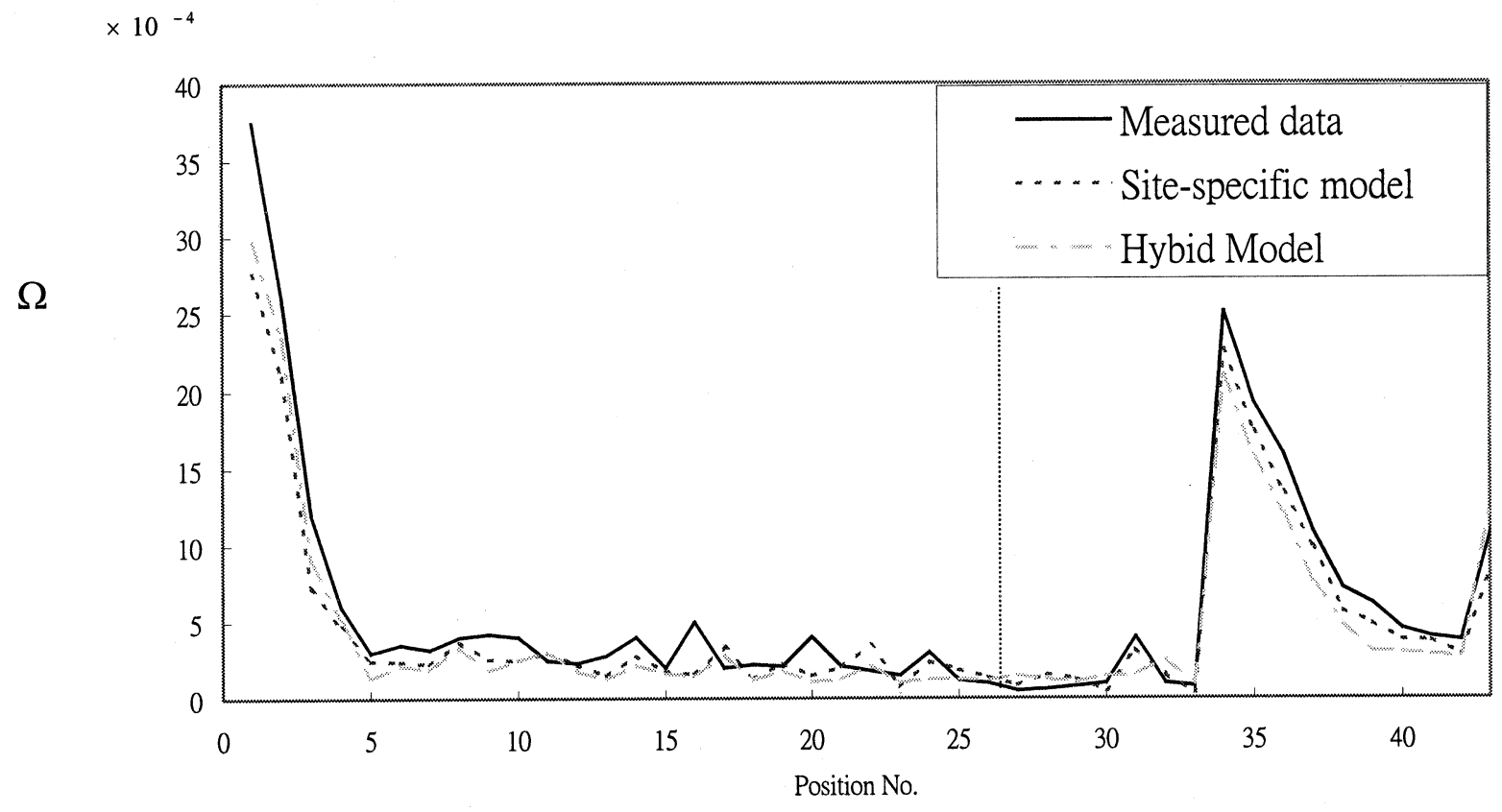

Fig. 10. The measured and computed $\Omega$ are summarized for the 43 receiving positions, where $\Omega$ represents the mean power. The measured data of position numbers greater than 26 are used for blind test.

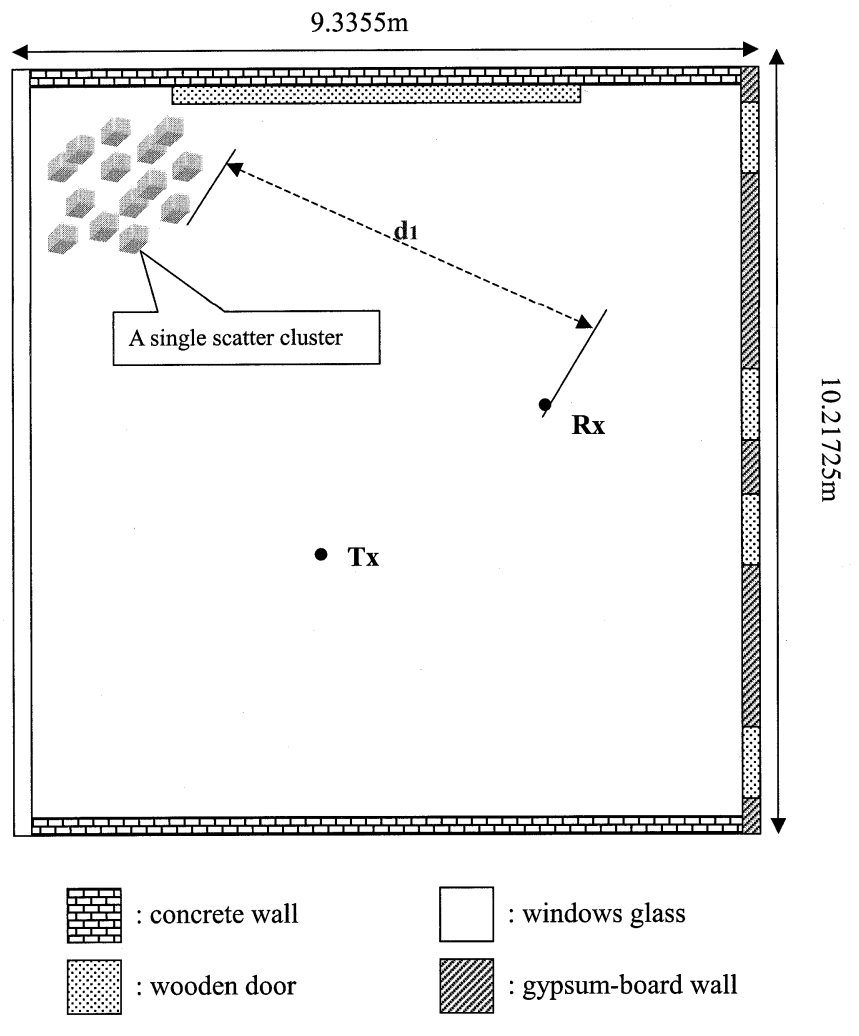

Fig. 11. Floor layout of site $\mathrm{G}$ (local scatterers). $d_{1}$ represents the distance between the $R x$ and scatterers.

shown in Fig. 11. In the measurement of signal strength distribution, the transmitting antenna is fixed and the receiving antenna is moving along a straight line. In Fig. 12, it is found that $r$ decreases, i.e., the cluttering strength decreases, as the receiving antenna moves away from the single scatterer cluster.
The light-cluttering situation happens when the distance between the receiving antenna and the scatterers is larger than 12 wavelengths. From the above discussion, it is found that the light-cluttering situation happens when the transmitting or the receiving antenna is some distance away from a single scatterer cluster, or when the reflectors have rough surfaces.

If there are many scatterer clusters around the transmitting or receiving antennas such as at sites $\mathrm{C}$ (a laboratory), $\mathrm{E}$ (a dormitory), and $\mathrm{F}$ (a computer room), then a heavy-cluttering situation will happen with large probability.

\section{Kind of Room}

We classify the propagation environment based on the kind of room. These include a corridor, a laboratory, a computer room, a dormitory, an auditorium, and an empty room. In Table II, it is observed that in a light-cluttering environment, radio propagates in an empty space such as in the corridor (Pos. no. 1-5), auditorium (Pos. no. 43), or an empty room (Pos. no. 6-12, 34-42). The heavy-cluttering situation happens when the room is crowded with a lot of scattering clusters such as in the laboratory (Pos. no. 13-22), dormitory (Pos. no. 23-26), or computer room (Pos. no. 27-33).

\section{D. $L O S / N L O S$}

From Table II, it is also found that $r$ distributes in the range of $0.35 \sim 0.75$ for NLOS environments and $0.25 \sim 0.35$ for LOS environments. In NLOS environments, $r$ may change in a wider range than in that of LOS environments, and classification based on LOS/NLOS may not be very effective.

Therefore, effects of the local scatterer clusters and the local reflectors with rough surface dominate the random fluctuation of the field strength. Their effects, quantified by the scattering factor $r$, are mainly dependent on the distance between the local 


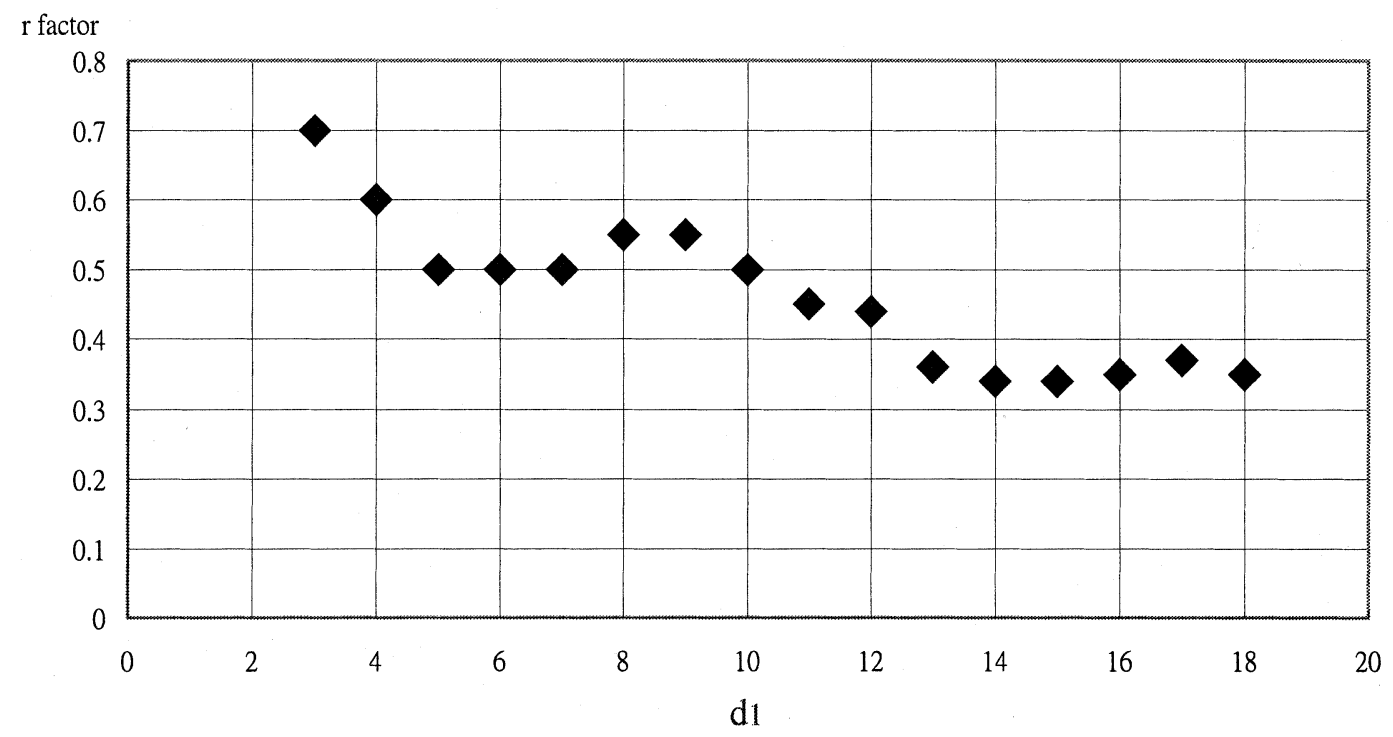

Fig. 12. Relative scattering factor $r$ versus $d_{1}$, where $d$ represents the distance between the receiver and the scatterer cluster.

TABLE II

RELATIVE SCATTERING FACTOR FOR 43 POSITIONS

\begin{tabular}{|c|c|c|c|}
\hline & Light-cluttering & & Heavy-cluttering \\
\hline Position No. & $r$ factor & Position No. & $r$ factor \\
\hline 1 & 0.28 & 13 & 0.7 \\
\hline 2 & 0.28 & 14 & 0.7 \\
\hline 3 & 0.26 & 15 & 0.3 \\
\hline 4 & 0.29 & 16 & 0.3 \\
\hline 5 & 0.29 & 17 & 0.28 \\
\hline 6 & 0.28 & 18 & 0.29 \\
\hline 7 & 0.27 & 19 & 0.3 \\
\hline 8 & 0.27 & 20 & 0.7 \\
\hline 9 & 0.3 & 21 & 0.72 \\
\hline 10 & 0.27 & 22 & 0.7 \\
\hline 11 & 0.29 & 23 & 0.69 \\
\hline 12 & 0.27 & 24 & 0.72 \\
\hline 34 & 0.3 & 25 & 0.68 \\
\hline 35 & 0.3 & 26 & 0.7 \\
\hline 36 & 0.3 & 27 & 0.71 \\
\hline 37 & 0.32 & 28 & 0.67 \\
\hline 38 & 0.35 & 29 & 0.71 \\
\hline 39 & 0.32 & 30 & 0.71 \\
\hline 40 & $\mathbf{0 . 3 1}$ & 31 & 0.65 \\
\hline 41 & 0.35 & 32 & 0.67 \\
\hline 42 & 0.35 & 33 & 0.26 \\
\hline 43 & 0.2 & & \\
\hline
\end{tabular}

cluster or the reflector and the transmitting/receiving antenna and number of local scattering clusters.

\section{CONCLUSION}

We found that the effects of the local scatterer clusters and the local reflectors with rough surface dominate the random fluctuation of the field strength, i.e., the small-scale fading. In this paper, we purpose a novel hybrid model to quantify their (clut- tering) effects, which uses a scattering factor $r$. With many measurements of 2.44-GHz radio at different sites, it is found that the factor $r$ is an effective index to describe the cluttering effect: 1) light-cluttering situation $r \leqq 0.35$ and 2) heavy-cluttering situation $r \geqq 0.65$. It is also found that there is a large probability that heavy-cluttering situations will happen when 1) there are many scatterer clusters around the transmitting and/or receiving antennas or 2) a single scatterer cluster or a reflector with rough surface is very close to (within 12 wavelengths) the transmitting and/or receiving antennas. The cluttering effect in the latter situation decreases when the transmitting or the receiving antenna is moved away from the single scattering object. The blind test finds that $r$ equals 0.3 and 0.7 for light-cluttering and heavy-cluttering environments, respectively, a reasonable choice. Notably, in a large open area, the optimum $r$ becomes smaller and equals 0.2 .

\section{REFERENCES}

[1] H. Hashemi, "The indoor radio propagation channel," Proc. IEEE, vol. 81, no. 7, pp. 943-968, 1993.

[2] D. Molkdar, "Review on radio propagation into and within buildings," Proc. Inst. Electr. Eng., pt. H, vol. 138, pp. 61-73, Feb. 1991.

[3] S. Y. Seidel and T. S. Rappaport, "Path loss prediction in multifloored buildings at $914 \mathrm{MHz}$," Electron. Lett., vol. 27, pp. 1384-1387, July 1991.

[4] A. J. Motley and J. M. P. Keenan, "Radio coverage in buildings," Br. Tel. Technol. J. (Special Issue Mobile Commun.), vol. 8, pp. 19-24, 1990.

[5] A. A. M. Saleh and R. A. Valenzuela, "A statistical model for indoor multipath propagation," IEEE J. Select. Areas Comm., vol. SAC-5, pp. 138-146, Feb. 1987.

[6] S. C. Kim, H. C. Bertoni, and M. Stern, "Pulse propagation characteristics at $2.44 \mathrm{GHz}$ inside buildings," IEEE Trans. Veh. Technol., vol. 45, no. 3, pp. 579-592, 1996.

[7] W. Honcharenko, H. L. Bertoni, J. L. Dailing, J. Qian, and H. D. Yee, "Mechanisms governing UHF propagation on single floors in modern office buildings," IEEE Trans. Veh. Technol., vol. 41, no. 4, pp. 496-504, 1992.

[8] S. Y. Seidel and T. S. Rappaport, "Site-specific propagation prediction for wireless in-building personal communication system design," IEEE Trans. Veh. Technol., vol. 43, no. 4, pp. 879-892, 1994.

[9] J. H. Tarng, W. R. Chang, and B. J. Hsu, "Three-dimensional modeling of $900-\mathrm{MHz}$ and $2.44-\mathrm{GHz}$ radio propagation in corridors," IEEE Trans. Veh. Technol., vol. 46, no. 2, pp. 519-527, 1997. 
[10] D. I. Laurenson, S. McLaughlin, and A. U. H. Sheikh, "Channel statistics analysis using a ray based approach," in PIMRC'94, vol. 4, 1994, pp. $1378-1382$.

[11] _ " "The application of ray tracing and the geometrical theory of diffraction to indoor channel modeling," IEEE Trans. Veh. Technol., vol. 44, no. 3, pp. 494-505, 1995 .

[12] M. Dottling, A. Jahn, H. Ernst, and S. Buonomo, "A versatile propagation channel simulator for land mobile satellite applications," IEEE Trans. Veh. Technol., pp. 213-217, 1998.

[13] _ - "Land mobile satellite propagation channel-A combined deterministic and statistical modeling approach," in Proc. 4th Eur. Conf. Sat. Comms. (ECSC4), 1997, pp. 182-187.

[14] A. Ishimaru, Electromagnetic Wave Propagation, Radiation, and Scattering. Englewood Cliffs, NJ: Prentice-Hall, 1991.

[15] E. N. Gilbert, "Energy reception for mobile radio," Bell Syst. Tech. J., vol. 44, no. 8, pp. 1779-1803, 1965.

[16] R. H. Clarke, "A statistical theory of mobile-radio reception," Bell Syst. Tech. J., vol. 47, pp. 957-1000, 1968.

[17] C. F. Yang, C. J. Ko, and C. J. Chen, "Measurement of the dielectric constants of the walls in building," in Proc. 1st Radio Science Symp., Kaohsiung, Taiwan, R.O.C., 1995, pp. 63-67.

[18] M. C. Lawton and J. P. McGeehan, "The application of a deterministic ray launching algorithm for the prediction of radio channel characteristic in small-cell environments," IEEE Trans. Veh. Technol., vol. 43, no. 4, pp. 966-969, 1994.

[19] J. E. Berg, R. Bowds, and F. Lotse, "Path loss and fading models for microcells at $900 \mathrm{MHz}$," in Proc. VTC, 1992, pp. 666-671.

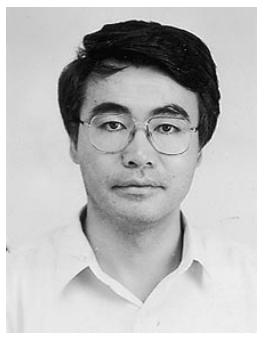

J. H. Tarng (S'85-M'86) received the B.S. degree in power mechanical engineering from National Tsin-Hua University, Hsin-Chu, Taiwan, R.O.C., in 1981 and the M.S. and Ph.D. degrees in electrical engineering from Pennsylvania State University, University Park, in 1988 and 1989, respectively.

$\mathrm{He}$ is a Professor in the Department of Communication Engineering, National Chiao-Tung University, Hsin-Chu. His professional interests include radio propagation modeling and measurement, radio allocation, radio network planning, smart antennas

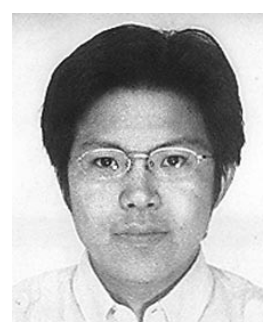

Wen-Shun Liu received the B.S. degree in chemical engineering from National Taiwan University, Taipei, Taiwan, R.O.C., in 1999 and the M.S. degree in communication engineering from National Chao Tung University, Hsin-Chu, Taiwan, in 2001.

$\mathrm{He}$ is currently an Engineer with the Chung Shan Institute of Science and Technology, Taoyuan. His interests include RF/analog circuit design and wireless communication.

Yeh-Fong Huang received the B.S. degree in electrical engineering from Yuan-Ze University, Taiwan, R.O.C. and the M.S. degree in communication engineering from National Chao Tung University, Hsin-Chu, Taiwan, in 1994 and 1999, respectively.

He is currently an Engineer in Chungwa Telecom Co., Ltd., Taoyuan, Taiwan. His research interests are in radio propagation modeling and cellular communication systems.

Jiunn-Ming Huang received the B.Ed. degree from the National Changhua University of Education, Changhua, Taiwan, R.O.C., in 1985, the M.S. degree in electrical engineering from Chung Cheng Institute of Technology, Taoyuan, Taiwan, in 1987, and the Ph.D. degree in communication engineering from National Chiao Tung University, Hsinchu, Taiwan, in 2000.

From 1987 to 1993, he was with Chung Shan Institute of Science and Technology working on antenna design and scattering measurement. From 1994 to 1998, he was a Consultant on mobile antenna design, amateur radio, and RF circuit design. From 1999 to 2001, he was with Taiwan Semiconductor Manufacturing Company working on RFIC plannar inductor, interconnect, and substrate noise characterization. His current interests include optimization theory in antenna and RFIC design, channel modeling, and signal integrity in VLSI. 